$\Rightarrow$ PROSTATE CANCER

\section{Mitochondrial metabolism: a target in AR-driven disease}

New data published in Nature Metabolism show that the androgen receptor (AR) transcriptionally regulates the mitochondrial pyruvate carrier (MPC). Inhibition of the MPC in preclinical models of AR-driven prostate cancer using a clinically viable molecule suppressed tumour growth; thus, targeting the MPC could be a therapeutic option for men with this disease.

Analysis of The Cancer Genome Atlas data for putative AR target genes involved in metabolism highlighted MPC2. MPC2 expression was elevated in prostate cancer tissue compared with benign tissue and men with high MPC2 tumour expression had reduced disease-free survival.

In prostate cancer cell lines, MPC subunit expression was increased in AR-positive, hormone-sensitive and castration-resistant cells, but was almost absent in AR-negative cells. MPC2 expression was increased by androgen treatment in AR-positive, hormone-sensitive prostate cancer cells, an effect that was blocked by treatment with enzalutamide. In vivo, castration significantly reduced MPC subunit expression initially; however,

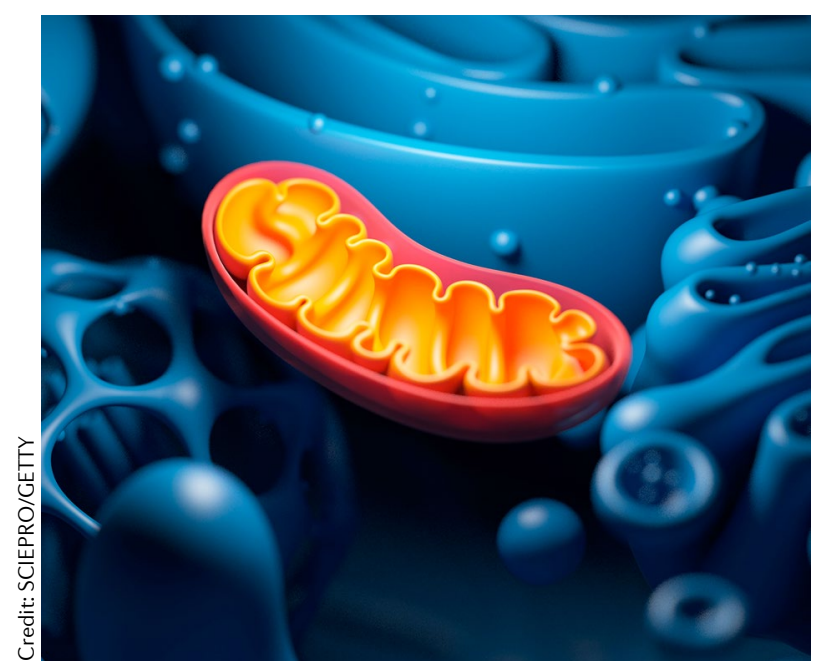

MPC expression re-emerged in castration-resistant tumours.

Analysis of transcription factor binding motifs identified two putative androgen response element half sites in the first intron of $M P C 2$. Recruitment of the AR to these sites was blocked by enzalutamide treatment and AR binding at the MPC2 locus was conserved in primary and castration-resistant prostate cancer.

Treatment of AR-dependent, hormone-responsive and castration-resistant prostate cancer cells with the MPC inhibitor UK5099 resulted in reduced proliferation. MPC inhibition also restricted maximal oxidative phosphorylation capacity and lipogenesis, and mitochondrial cristae were swollen in hormone-sensitive cells. In castration-resistant cells, MPC inhibition resulted in restricted basal and maximal oxygen consumption rate and an increased rate of extracellular acidification. Effects of UK5099 on proliferation were absent or minimal in AR-negative cells lacking MPC expression.

MPC inhibition resulted in considerable disruption of metabolic homeostasis, affecting intracellular metabolite pools and reducing potential, ATP content and antioxidant content. Tracing experiments combined with MPC inhibition showed that glucose is the primary carbon source for the tricarboxylic acid (TCA) cycle and that the MPC is crucial in maintaining metabolic outputs of the TCA cycle in castration-resistant cells. Results from reverse-phase protein array and RNA sequencing suggested that MPC inhibition expedites integrated stress response (ISR) activation, delaying cell cycle progression.

During MPC inhibition, glutamine restriction increased TCA metabolite depletion and ATF4 activation. Cells grown in the absence of glutamine or treated with glutamine inhibitors were sensitized to MPC inhibition. AR-positive cells (which express MPC) grew in the absence of glutamine but AR-negative cells (which lack MPC) did not.

In vivo, MPC inhibition with UK5099 suppressed tumour growth in AR-positive, castration-resistant xenografts. Additionally, oral treatment with the clinically viable, small-molecule MPC inhibitor MSDC0160 suppressed growth in these xenografts and also in AR-positive, hormone-sensitive tumours. MSDC0160-treated tumours showed evidence of ISR activation. Mice given MSDC0160 did not display any signs of treatmentassociated toxic effects.

"Our approach is novel in that we are attempting to target a critical functional output of AR transcriptional activity (MPC activity) rather than AR transcription itself, which is particularly important as the majority of men who die of prostate cancer die of AR-positive castration-resistant disease," David Bader and Sean McGuire, corresponding authors on the paper, tell Nature Reviews Urology. "Based on our current experiments, this disease remains sensitive to MPC inhibition," they continue. "We identify ATF4 and ISR signalling as the predominant mechanisms responsible for preventing cell cycle progression. Furthermore, we think that combinatorial approaches to suppress the MPC and restrict glutamine availability hold therapeutic promise for the management of prostate tumours," they explain. "By using an orally bioavailable and clinically viable small molecule (MSDC0160), our results have immediate implications for clinical translation."

Louise Stone

ORIGINAL ARTICLE Bader, D. A. et al.

Mitochondrial pyruvate import is a metabolic vulnerability in androgen receptor-driven prostate cancer. Nat. Metab. https://doi.org/10.1038/ 542255-018-0002-y (2018) 